\title{
Facilitating Remote Design Thinking Workshops in Healthcare: the Case of Contouring in Radiation Oncology
}

\author{
Matin Yarmand \\ Computer Science and Engineering, \\ UC San Diego \\ La Jolla, CA, USA
}

\author{
Chen Chen \\ Computer Science and Engineering, \\ UC San Diego \\ La Jolla, CA, USA
}

\author{
Danilo Gasques \\ Computer Science and Engineering, \\ UC San Diego \\ La Jolla, CA, USA
}

\author{
James D. Murphy \\ Radiation Medicine and Applied \\ Sciences, UC San Diego \\ La Jolla, CA, USA
}

\author{
Nadir Weibel \\ Computer Science and Engineering, \\ UC San Diego \\ La Jolla, CA, USA
}

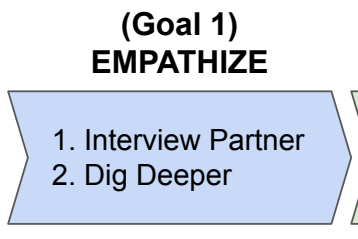

(Goal 2) DEFINE

3. Capture Findings

4. Define Problem

Statement
(Goal 3)

IDEATE

5. Sketch to Ideate

6. Share Solutions \& Capture Feedback
(Goal 4) PROTOTYPE

7. Reflect \& Generate a New Solution
(Goal 5)

TEST

8. Build
9. Group Gather \&
Get Feedback

Figure 1: The 5 goals and 9 steps of our remote design thinking workshops based on guidelines of the Wallet project [2]. Due to time constraints, we combined the last 2 steps of the Wallet project (i.e., Share Solution and Group Debrief) into step 9: participants shared solutions with the entire group and initiated discussions about strengths and weaknesses of their prototypes.

\begin{abstract}
Co-location matters, especially when running collaborative design thinking workshops. What if participation cannot occur in person? How can we conduct these workshops remotely? Based on guidelines of the Wallet project that provides a framework for in-person design thinking workshops, we present the first experiences of facilitating 2 remote design workshops with radiation oncology faculty and residents. We report the logistics and tool support decisions for the first workshop, evaluate and adjust our approach for the second workshop, and present the 3 lessons learned: incorporating schedule flexibility, prioritizing technology familiarity, and integrating communication channels.
\end{abstract}

\section{CCS CONCEPTS}

- Human-centered computing $\rightarrow$ Interaction design process and methods; User studies.

\section{KEYWORDS}

Remote Design Thinking Workshop, HCI Design Methodology

Permission to make digital or hard copies of part or all of this work for personal or classroom use is granted without fee provided that copies are not made or distributed for profit or commercial advantage and that copies bear this notice and the full citation on the first page. Copyrights for third-party components of this work must be honored.

For all other uses, contact the owner/author(s).

CHI '21 Extended Abstracts, May 8-13, 2021, Yokohama, Japan

(c) 2021 Copyright held by the owner/author(s)

ACM ISBN 978-1-4503-8095-9/21/05.

https://doi.org/10.1145/3411763.3443445
ACM Reference Format:

Matin Yarmand, Chen Chen, Danilo Gasques, James D. Murphy, and Nadir Weibel. 2021. Facilitating Remote Design Thinking Workshops in Healthcare: the Case of Contouring in Radiation Oncology. In CHI Conference on Human Factors in Computing Systems Extended Abstracts (CHI '21 Extended Abstracts), May 8-13, 2021, Yokohama, Japan. ACM, New York, NY, USA, 5 pages. https://doi.org/10.1145/3411763.3443445

\section{INTRODUCTION}

Design thinking workshops provide a human-centered framework for problem solving [4], and foster exploring needs and ideas for a particular group of users [5]. The iconic Wallet project [2], proposed by Stanford's School of Design, offers a fast-paced template for guiding workshop participants through the entire design thinking process: it empowers people to collaboratively gain empathy, define their needs, generate ideas, prototype solutions, and receive feedback on their prototypes. This project was originally designed for co-located settings where nearby participants can pair up for discussions, sketch ideas on paper, and create a physical prototype.

While prior interpretations of this workshop were conducted in person [3,6-8], remote design thinking workshops can be facilitated when co-location gatherings are not possible, such as during pandemics when social distancing practices are required. We report first experiences of facilitating 2 remote design thinking workshops with radiation oncologists about contouring delineation. We discuss the challenges we faced in the first workshop and the adjustments we made for the second workshop, such as the choice of tools for note-taking (i.e., Google Doc vs Google Slides) and prototyping (i.e., Lucidchart vs Google Slides). Lastly, we share 3 lessons learned: incorporating flexibility, prioritizing technology familiarity over sophistication, and integrating communication channels. 


\section{METHODS AND FINDINGS: TWO DESIGN THINKING WORKSHOPS}

We first introduce the motivations and goals for our design thinking workshops and then describe the implementation details.

\subsection{Motivations and Goals}

In the field of radiation oncology, treating patients with safe and effective radiation therapy depends on the precise identification of tumour tissues. Contouring refers to the practice of outlining cancer and normal tissues on medical images, such as Positron Emission Tomography (PET) and Computed Tomography (CT). Contouring education occurs during the radiation oncology residency when residents learn from the attending physicians. This training follows the apprenticeship model where the resident watches as the faculty contours a case, attempts to replicate similar contours on new cases, and receives feedback from their faculty. This process takes a long time, especially between the resident submitting their contours and receiving feedback from their faculty. Besides, viewing the contoured cases can only occur on special computer softwares (e.g., MIM) which might not always be accessible.

To re-imagine the learning and teaching processes of contouring education, we organized 2 design thinking workshops and invited 4 faculty $(1 \mathrm{~F}, 3 \mathrm{M}$; $32-39$ years old) and 6 residents $(2 \mathrm{~F}, 4 \mathrm{M} ; 28-35$ years old) from the Department of Radiation Medicine at University of California San Diego. Based on the guidelines of the Wallet project, we guided the participants to define their contouring needs and explore solutions. The main goal of the workshops was to design on-demand, contouring feedback interfaces on mobile devices. The focus of the first workshop with the faculty was the content of the feedback (i.e., what should be presented), and the second workshop with the residents was focused on how feedback should be presented.

\subsection{Logistic Management}

Moderators The organizing team consisted of 4 and 5 moderators for the first and second workshops, respectively. The first author was responsible for leading the workshops and providing instructions. The second author assigned participants to discussion groups and updated their worksheets during the sessions. The third and fourth (as well as the first) authors joined discussion groups to monitor the progress of participants and provide assistance if needed. We also invited one senior faculty from the Department of Radiation Medicine to drive the group discussion in the final debrief step.

Workflow Based on guidelines of the Wallet project [2], we facilitated remote design thinking workshops in radiation oncology. Despite participants' prior commitment to attend for the entire 2 hour duration of the workshop, unexpected personal and technology circumstances delayed the progress of the sessions. In the first workshop, 3 participants (75\%) left their workspaces multiple times due to personal affairs (e.g., receiving a package delivery). In the second workshop, one participant's microphone malfunctioned during step 6 which hindered the pair's discussion. To address these delays, we needed to allocate more time to let the affected participants catch up with the rest of the group which led to insufficient time at the end. As such, we merged the last two steps of the Wallet project (i.e., sharing solution/exchanging feedback and group gather/debrief) into one final step: after collaboratively building a digital prototype, we invited pairs of participants to share their solutions with the entire group and collectively discuss points of strengths and weaknesses. The 5 goals and 9 steps of our project are demonstrated in Figure 1.

\subsection{Tool Support}

Video Conferencing We used Zoom ${ }^{1}$ as the main video conferencing tool among the organizers and participants, primarily because of the following collaborative features:

- Screen Sharing was used to deliver the instructions for every step similar to a projector in co-located settings. As suggested in the Wallet project, we also played upbeat music during the individual and pair steps. To do so, one member of the organizing team shared their screen and enabled audio.

- Breakout Rooms facilitated simultaneous pair activities for steps 1, 2, 6, and 8 of the workshop (i.e., Interview, Dig Deeper, Share solutions \& Capture Feedback, and Build) where participants discussed ideas without interfering with other pairs. In addition to 2 pre-assigned participants, one researcher joined each breakout room to record the discussion and clarify instructions as needed. The researchers kept their cameras and microphones turned off to minimize distractions. One researcher stayed in the main meeting room and broadcasted time reminders in the chat and brought everyone back at the end of each step.

Note-taking Similar to the Wallet project, we provided worksheets to participants for their notes and sketches. Due to the challenges of drawing sketches using mouse in a remote setting, the participants could choose to describe their sketches instead. We provided the worksheet pages periodically during the workshops: this encouraged participants to focus on the current task without getting distracted by the next steps.

During the first workshop, we used Google $\operatorname{Doc}^{2}$ and inserted drawing boxes on the blank spaces of the worksheet to facilitate both writing and sketching. One researcher was responsible to add new pages to all participants' worksheets while another presented instructions. During the first workshop, we realized that formatting created delays in updating the worksheets as adding new pages moved around the previous content. We also noticed that one participant (25\%) chose not to edit the drawing boxes, and instead leave comments.

Due to formatting obstacles, we used Google Slides ${ }^{3}$ as the notetaking tool in the second workshop, designating each slide to a page of the worksheet. In terms of note-taking, Google Slides fostered three improvements compared to Google Doc. First, it allowed us to copy slides faster and more consistently, since duplicating a slide did not require object selection and re-formatting. Second, Google Slides enhanced readability and aesthetics of the text boxes, as shown in Figure 2. Third, manipulating the text boxes on Google Slides were interactionally less demanding (i.e., Google Slides participants to

\footnotetext{
${ }^{1}$ Zoom: https://zoom.us

${ }^{2}$ Google Doc: https://doc.google.com

${ }^{3}$ Google Slides: https://slides.google.com
} 
Ideate: generate alternatives to test.

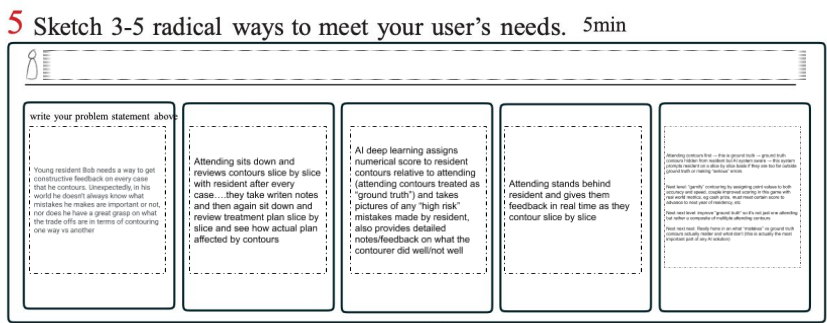

6 Share your solutions \& capture feedback. 10 min $(2$ sessions $\mathrm{x} 5$ minutes each) Switch roles \&

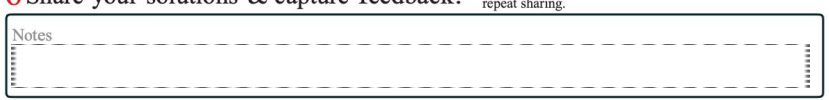

(a) Workshop I: Note-taking on Google Doc Ideate: generate alternatives to test.

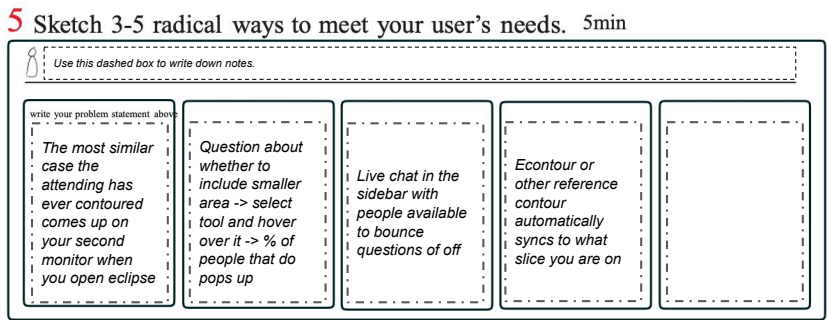

6 Share your solutions \& capture feedback. $10 \mathrm{~min}$ (2 sessions $\times 5$ minutes each) Switch roles \& repeat sharing.

Live demos are useful but only as a $1 x$ exercise

(b) Workshop II: Note-taking on Google Slides

Figure 2: Two sample worksheets from the first and second workshops on Google Doc and Google Slides respectively. The participants' responses on Google Slides (i.e., 2b) were easier to read, and interactionally less demanding.

edited the text boxes by double clicking on them, while Google Doc participants needed to double click to activate a pop-up area for editing, make their changes, and then click the SAVE button).

Collaborative Prototyping At step 8 (i.e., Build), participants collaboratively built a digital prototype for their solution. In pairs, participants were allocated 25 minutes to design a contouring feedback interface on a mobile device. Similar to previous steps, one researcher attended each breakout room to provide technical support, if needed.

We used Lucidchart ${ }^{4}$ as the prototyping tool of the first workshop because of its robust diagraming tools and collaborative features: it contains many quality logos, shapes, and images that can be searched and incorporated onto the main canvas. It also shares real-time cursor movements to further enhance the collaborative feel of the process. In the beginning, we offered a short tutorial of the main features of the software to enhance familiarity. Figure 3a displays our setup of Lucidchart.

$\overline{{ }^{4} \text { Lucidchart: https://lucidchart.com }}$

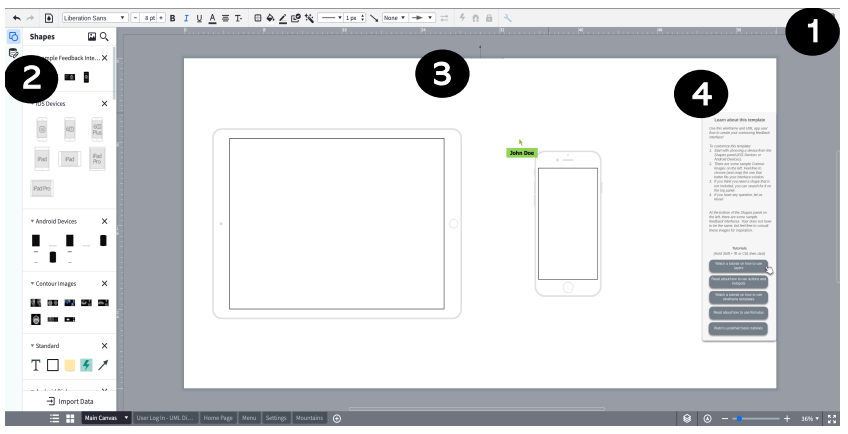

(a) Workshop I: Lucidchart Interface

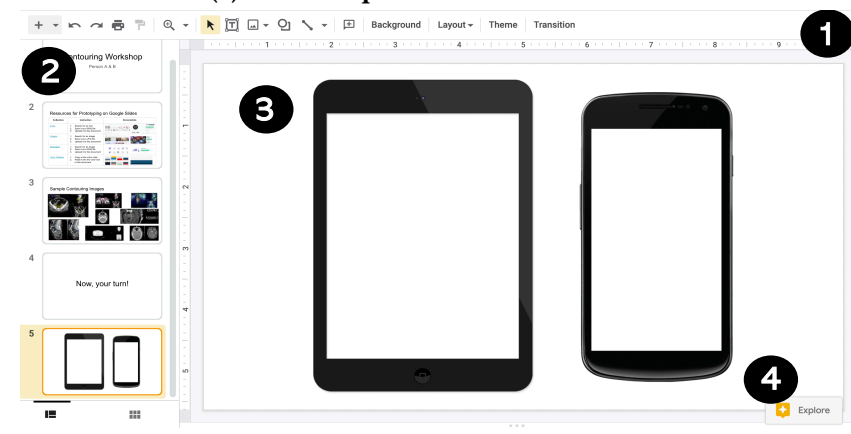

(b) Workshop II: Google Slides Interface

Figure 3: The set-up prototyping interfaces of (a) Lucidchart and (b) Google Slides. While Lucidchart offers extensive features, Google Slides is a more familiar tool. Each interface contains: (1) tool bar with general functionalities such as shapes and fonts, (2) provided components repository including sample medical images, (3) main canvas for prototyping, and (4) tutorial resources in the form of relevant links (i.e., 3a) and automatic search (i.e., 3b).

Participants' lack of familiarity with Lucidchart caused difficulties during the prototyping step. 1 pair (out of 2) faced sign-up issues which significantly delayed their prototyping. Eventually, the assigned researcher had to share their screen while the participants described their solution. While the other pair successfully signed up, they did not fully collaborate: one person - who was confused with the software - stopped manipulating the features shortly after they started, and instead, verbally provided suggestions to the other participant.

To ease the complications of signing up and using the features of Lucidchart, we incorporated Google Slides in the second workshop. Since Google services were commonly used in the university, participants easily signed up into their prototyping space. They were also familiar with Google Slides' features which greatly improved how they collaborated on their prototyping tasks; the participants inserted shapes, imported their own pictures, and added texts. Figure 3 depicts the two prototyping interfaces.

Moderator Communications We used Slack ${ }^{5}$ to address communication needs among the organizers, since Zoom lacked any

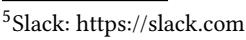


subgroup messaging feature. To avoid broadcasting disruptive notification sounds while screen sharing, we accessed Slack via our mobile devices. One of the challenges of virtual workshops was understanding the crowd and making in-time adjustments, especially when participant pairs were in disconnected breakout rooms. We communicated the progress of the pairs via Slack and adjusted the allocated time if needed, such as when some participant's technical malfunctions delayed their discussion. We noticed, however, that the separation of the organizational communication (i.e., Slack) and the main workshop (i.e., Zoom) caused delays in response. Some members of the research team did not see the messages until later steps which hindered prompt planning.

\section{DISCUSSIONS: LESSONS LEARNED}

\subsection{Introducing Flexibility}

One challenge in designing remote workshops is to synchronize the progress of all participants. This is particularly important in design thinking workshops which people learn, ideate, and prototype together: out of sync participants cannot collaborate productively with each other. As previously mentioned, the participants encountered environmental (e.g., package delivery) and technical (e.g., microphone malfunctions) obstacles to stay engaged with the workshop. This is more prominent in online workshops where there is less control over remote attendance.

To address this challenge, we recommend incorporating more flexibility in remote design workshops. Organizers can add "whitespaces" to the workshop schedule to account for potential attendance inconsistencies. So if someone has to momentarily step out of their workspace, more time can be allocated without jeopardizing the later steps. Another solution is to introduce more asynchronicity into the implementation of a design thinking workshop. The current design of the Wallet project revolves around same-time interactions with the same set of pairs on the same set of steps. We believe that a robust remote workshop can dynamically pair participants based on their level of progress, meaning that "slower" participants can be matched ad-hoc while the "faster" participants progress to the later steps. This also requires decoupling of steps such that any two participants can collaborate on any step without having worked together beforehand.

\subsection{Using Familiar Technology}

When designing the workshops, we noticed a trade-off between software sophistication and user familiarity. Unlike Google Slides, LucidChart contained a large collection of professional-looking images and icons, as well as cursor sharing among users to enhance collaboration. While these affordances have the potential to improve group prototyping experience, the participants' lack of familiarity with the features posed a significant obstacle in fast-paced design thinking workshops. We also noticed that the participants did not take advantage of the provided tutorials during the prototyping step. We suspect that it might have been due to insufficient time and the complexity of the resources.

We recommend workshop organizers to consider participants' familiarity for tool selection. Tool familiarity can boost task performance, because unfamiliar tools require new skills and patterns of interaction $[1,9]$. This does not undermine the potential benefits of more sophisticated tools, but highlights the importance of building familiarity by the time of the workshop. As such, we recommend two principles for designing and distribution of tutorial resources: timing and simplicity. If participants lack previous exposure to particular tools, we recommend organizers to send tutorial resources ahead of time and encourage exploration. Also, simple and short tutorials can encourage engagement with the content before and during the workshop.

\subsection{Integrating Communication Channels}

We used Slack as a separate messaging system among the research team. While the separation lessened disruptions into the main workshop, we noticed that a major downside of this separation was potential response delays as members needed to periodically check multiple devices (i.e., Zoom on computer and Slack on mobile devices). Communication among the organizing team was crucial for running remote workshops. Compared to in-person settings, understanding the crowd in a remote environment was challenging, especially during separated breakout rooms. Besides, the remote attendance created inconsistencies in people's participation which required ad-hoc time adjustments.

To address these challenges, one solution is to integrate the communication channels non-obtrusively: both organizer-participant and organizer-organizer communication channels can be accessed on the same platform, yet the visual and auditory notification are distributed without disruptions in the workflow. We envision Slack's threaded messaging and notification system within Zoom's interface as a viable solution for the difficulties that we faced.

\section{CONCLUSIONS AND FUTURE WORK}

This case study presents our experience of running remote design thinking workshops with radiation oncology residents and faculty. We experimented with note-taking and collaborative prototyping technologies and offer the following recommendations for future remote workshop organizers:

(1) Plan ahead for participation inconsistencies. Design a flexible schedule and incorporate asynchronicity.

(2) Lean towards familiar prototyping technologies. If you choose to incorporate a less familiar, but more sophisticated software, provide simple tutorials ahead of time.

(3) Select a video conferencing tool that offers integrated group messaging for organizational communication.

We hope that the presented lessons can help the HCI community design remote workshops not only during health crises, but also as a way to gather a more global and diverse set of perspectives. Future work can further explore remote design thinking workshops, present organizational strategies, and re-structure the guidelines of the Wallet project to better fit remote settings.

\section{ACKNOWLEDGMENTS}

We thank the anonymous reviewers for their insightful feedback. This work was supported in part by the Agency for Healthcare Research and Quality (AHRQ). 


\section{REFERENCES}

[1] Alan R Dennis and Monica J Garfield. 2003. The adoption and use of GSS in project teams: Toward more participative processes and outcomes. MIS quarterly (2003), 289-323.

[2] Stanford University Design School. 2016. The wallet project. https://dschool stanford.edu/resources/the-gift-giving-project

[3] Kirsten H Dodson, Kerry E Patterson, and Joseph B Tipton. 2017. Work-inProgress-Emphasizing Human-Centered Design in the Freshman Year through an Interactive Engineering Design Process Experience. ASEE First Year Engineering Experience Conference Proceedings.

[4] Lucy Kimbell. 2011. Rethinking design thinking: Part I. Design and Culture 3, 3 (2011), 285-306.

[5] Tilmann Lindberg, Christine Noweski, and Christoph Meinel. 2010. Evolving discourses on design thinking: how design cognition inspires meta-disciplinary creative collaboration. Technoetic Arts: A Journal of Speculative Research 8, 1 (2010)

[6] Nathalia Peixoto, Jennifer Suh, Padmanabhan Seshaiyer, KW Lee, Yunsuk Jung, and Daniel Suh. 2014. An international collaboration to cultivate global innovators. In International Conference on Engineering Education (ICEE'14), Riga, Latvia.

[7] Yen Dieu Pham, Davide Fucci, and Walid Maalej. 2018. A first implementation of a design thinking workshop during a mobile app development course project. In Proceedings of the 2nd International Workshop on Software Engineering Education for Millennials. 56-63.

[8] Katherine Steele, Brianna Blaser, and Maya Cakmak. 2018. Accessible making: Designing makerspaces for accessibility. International fournal of Designs for Learning 9, 1 (2018), 114-121.

[9] John D Wells, William L Fuerst, and Jonathan W Palmer. 2005. Designing consumer interfaces for experiential tasks: an empirical investigation. European fournal of Information Systems 14, 3 (2005), 273-287. 